\title{
Improving Music Recommendation Using Distributed Representation
}

\author{
Dongjing Wang, Shuiguang Deng* \\ College of Computer Science \\ and Technology, Zhejiang University \\ Hangzhou, Zhejiang, China \\ \{tokyo1, dengsg\}@zju.edu.cn
}

\author{
Songguo Liu \\ Hangzhou National E-commerce \\ Product Quality Monitoring and \\ Management Center, China \\ Sgliu@zju.edu.cn
}

\author{
Guandong Xu \\ Advanced Analytics Institute, \\ University of Technology Sydney \\ Sydney, Australia \\ Guandong.xu@uts.edu.au
}

\begin{abstract}
In this paper, a music recommendation approach based on distributed representation is presented. The proposed approach firstly learns the distributed representations of music pieces and acquires users' preferences from listening records. Then, it recommends appropriate music pieces whose distributed representations are in accordance with target users' preferences. Experiments on a real world dataset demonstrate that the proposed approach outperforms the state-of-the-art methods.
\end{abstract}

\section{Keywords}

music recommendation; distributed representation

\section{INTRODUCTION}

Nowadays, digital music market is growing rapidly due to the prevalence of mobile devices and advance in the Internet technology. It is more important than ever to help people find the interested music pieces from massive music contents available on the Internet. How to extract the feature of music and incorporate them into music recommendation is still a challenging task. To address this problem, we present a music recommendation approach based on distributed representation. Firstly, the proposed approach learns the distributed representations (vectors in real-valued, low-dimensional space) of music pieces from all users' historical listening records. Then, it infers users' music preferences from their listening records with these distributed representations. Finally, our approach recommends appropriate music pieces according to target users' preferences to satisfy their requirements.

\section{PROPOSED APPROACH}

Music recommendation is to find music pieces that the target user would probably enjoy. Formally, let $U=\left\{u_{1}, u_{2}, \ldots, u_{|U|}\right\}$ be the user set and $M=\left\{m_{1}, m_{2}, \ldots, m_{|M|}\right\}$ be the music set. For each user $u$, his/her historical listening record is denoted as $H^{u}=\left\{m_{1}^{u}, m_{2}^{u}, \ldots, m_{\left|H^{u}\right|}^{u}\right\}$, where $m_{i}^{u} \in M$. Music in $H^{u}$ are sorted according to the corresponding playing time. Then, our

*The corresponding author is Shuiguang Deng and this work was supported in part by the National Key Technology Research and Development Program of China under Grant 2014BAD10B02.

Copyright is held by the author/owner(s).

WWW'16 Companion, April 11-15, 2016, Montréal, Québec, Canada. ACM 978-1-4503-4144-8/16/04.

DOI: http://dx.doi.org/10.1145/2872518.2889399 task specifies to be seeking for music that user $u$ may enjoy given his/her listening record $H^{u}$. To address this task, we propose a music recommendation approach, which consists of three steps: distributed representation learning, users' preferences acquisition, and recommendation.

Firstly, we propose the music2vec model to learn the distributed representations of all music pieces. Specifically, the music2vec model adopt a skip-gram model [1], which is much more efficient as well as memory-saving than other approaches, to learn the distributed representation by maximizing the objective function over music sequences in all users' listening records. The underlying idea of music2vec is that similar music pieces should have similar contexts. Formally, the objective function is defined as follows:

$$
\mathcal{L}=\sum_{u \in U} \sum_{m_{i}^{u} \in H^{u}} \sum_{-c \leq j \leq c, j \neq 0} \log p\left(m_{i+j}^{u} \mid m_{i}^{u}\right)
$$

where $c$ is the length of the context window. $p\left(m_{i+j}^{u} \mid m_{i}^{u}\right)$ represents the probability of observing a neighbor music piece

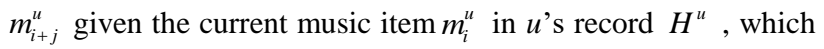
is formally defined using the soft-max function as follows:

$$
p\left(m_{i+j}^{u} \mid m_{i}^{u}\right)=\exp \left(\mathbf{v}_{m_{i}^{u}}^{T} \cdot \mathbf{v}_{m_{i+j}^{u}}^{\prime}\right) / \sum_{m \in M} \exp \left(\mathbf{v}_{m_{i}^{u}}^{T} \cdot \mathbf{v}_{m}^{\prime}\right)
$$

where $\mathbf{v}_{m}$ and $\mathbf{v}_{m}^{\prime}$ are the input and output distributed representations of music $m$, respectively. In the learning phase, we need to maximize the objective function defined in Equation 1 over all users' historical listening records. However, the complexity of computing corresponding soft-max function defined in Equation 2 is proportional to the music set size, which can reach millions easily. In this paper, we adopt negative sampling [1] to increase computation efficiency by generating a few noise samples for each input music to estimate the target music. Therefore, the training time yields linear scale to the number of noise samples. Finally, the distributed representations of all music pieces can be obtained.

Then, music preference of the target user is inferred from his/her historical listening record using the following formula:

$$
\mathbf{p}_{u}=\frac{1}{\left|H^{u}\right|} \sum_{m_{i}^{u} \in H^{u}} \mathbf{v}_{m_{i}^{u}}
$$

where $\mathbf{v}_{m_{i}^{u}}$ is the learned distributed representation of music $m_{i}^{u}$ using music2vec model.

Finally, we propose a music recommendation method which can recommend music pieces appropriate music pieces whose distributed representations are in accordance with target users' 
preferences. Specifically, the predicted interest $p i$ of the target user $u$ in music piece $m$ is the cosine similarity between $u$ 's music preference $\mathbf{p}_{u}$ and $m$ 's distributed representation $\mathbf{v}_{m}$, which is defined as follows:

$$
\operatorname{pi}\left(m \mid u, \mathbf{p}_{u}\right)=\cos \left(\mathbf{p}_{u}, \mathbf{v}_{m}\right)
$$

Therefore, the ranking of music pieces $>_{u, \mathbf{p}_{u}}$ in our approach is defined as

$$
m_{i}>_{u, \mathbf{p}_{u}} m_{i}^{\prime}: \Leftrightarrow \operatorname{pi}\left(m_{i} \mid u, \mathbf{p}^{u}\right)>\operatorname{pi}\left(m_{i}^{\prime} \mid u, \mathbf{p}^{u}\right)
$$

We then can recommend the music pieces with high ranking scores (similar to user's musical preference) to the target user.

\section{EXPERIMENTS}

The experiments consist of two parts: evaluation of music2vec and the comparison of the proposed approach with baselines.

Firstly, we illustrate the effect of music2vec model by visualizing similarity among music pieces given in Table 1 . As shown in Figure 1, music pieces with similar styles, such as singers, tags, and genres, have similar distributed representations. For example, "Summer" and "Moonlit Sea of Clouds", which have the same genre and player, do lie nearby in the real-valued distributed representation space. Besides, neither of these two music pieces has similar distributed representations with the other music pieces in Table 1. Therefore, the learned distributed representations with music2vec capture useful features effectively and depict music pieces well.

Table 1. Basic information of music examples

\begin{tabular}{|c|l|l|}
\hline No & \multicolumn{1}{|c|}{ Name-Singer } & \multicolumn{1}{c|}{ Tags } \\
\hline 1 & Hero-Mariah Carey & pop, female vocalists, 90s, ballad \\
\hline 2 & $\begin{array}{l}\text { Without You-Mariah } \\
\text { Carey }\end{array}$ & pop, female vocalists, soul, love \\
\hline 3 & Drowning-Backstreet Boys & pop, boy bands, ballad \\
\hline 4 & My Love-Westlife & pop, boy bands, Irish \\
\hline 5 & Don't Cry-Guns N' Roses & classic rock, hard rock, ballad \\
\hline 6 & Hotel California-Eagles & classic rock, rock, 70s \\
\hline 7 & Fall Again-Kenny G & smooth jazz, R\&B, Soul \\
\hline 8 & Heart and Soul-Kenny G & smooth jazz, Rhythm and blues \\
\hline 9 & Summer-Joe Hisaishi & $\begin{array}{l}\text { sound track, Japanese, anime, } \\
\text { instrumental, classical }\end{array}$ \\
\hline 10 & $\begin{array}{l}\text { Moonlit Sea of Clouds-Joe } \\
\text { Hisaishi }\end{array}$ & $\begin{array}{l}\text { sound track, Japanese, anime, } \\
\text { instrumental, classical }\end{array}$ \\
\hline
\end{tabular}

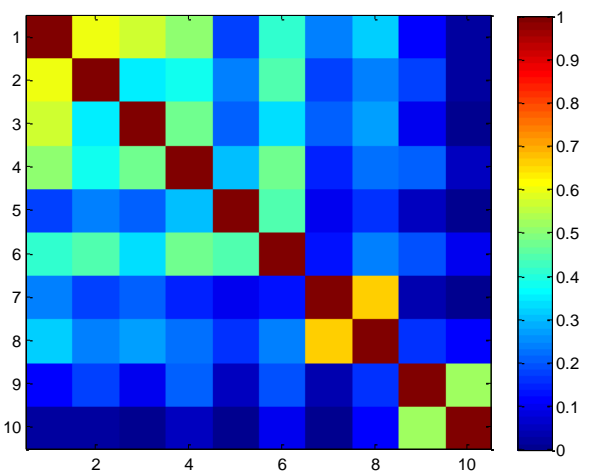

Figure 1. Similarity visualization of music examples with distributed representations

Then, we compare the proposed method with three state-ofthe-art recommendation algorithms, including Bayesian Personalized Ranking (BPR) [2], FISMauc (FISM) [3], and user based collaborative filtering method (UserKNN) [4] on a real world dataset collected from Xiami Music (http://www.xiami.com/). From the comparison results in Table 2 , we can see that our approach outperforms baselines in terms of F1 score and hitrate. Taking the F1 score as an example, when compared with BPR, FISM, and UserKNN with the recommending number being 10 , the relative performance improvement achieved by the proposed approach is around $33.3 \%, 20.7 \%$, and $42.2 \%$, respectively. The improvements indicate that our approach is more effective than baselines in acquiring users' preference and assisting music recommendation.

In conclusion, the proposed approach can effectively recommend music pieces appropriate for target users and satisfy their preferences well.

Table 2. Comparisons with baselines

\begin{tabular}{|c|c|c|c|c|}
\hline \multirow{2}{*}{ Methods } & \multicolumn{2}{|c|}{ F1, \% } & \multicolumn{2}{c|}{ Hitrate, \% } \\
\cline { 2 - 5 } & $@ \mathbf{1 0}$ & @ 20 & @ 10 & @ 20 \\
\hline Our approach & $\mathbf{7 . 8 8}$ & $\mathbf{9 . 2 3}$ & $\mathbf{3 6 . 6 5}$ & $\mathbf{4 3 . 1 4}$ \\
\hline BPR & 5.91 & 7.13 & 26.96 & 30.21 \\
\hline FISM & 6.53 & 7.50 & 29.68 & 33.52 \\
\hline UserKNN & 5.54 & 6.34 & 22.72 & 25.61 \\
\hline
\end{tabular}

\section{CONCLUSIONS AND FUTURE WORK}

We present a music recommendation approach, which learns the distributed representations of music pieces from users' historical listening records, and utilizes these distributed representations to acquire users' music preferences and recommend appropriate music pieces. Experimental results show the effectiveness of the proposed approach. There are two possible future directions. Firstly, we plan to combine the distributed representation with more advanced recommendation techniques [5, 6], to further improve the performance. Secondly, we will try to evaluate our approach by online experiments.

\section{REFERENCES}

[1] T. Mikolov, I. Sutskever, K. Chen, G.S. Corrado, and J. Dean, Distributed representations of words and phrases and their compositionality. In Advances in Neural Information Processing Systems, 3111-3119, 2013.

[2] S. Rendle, C. Freudenthaler, Z. Gantner, and L. SchmidtThieme, BPR: Bayesian personalized ranking from implicit feedback. In Proceedings of the 25th Conference on Uncertainty in Artificial Intelligence, 452-461, 2009.

[3] S. Kabbur, X. Ning, and G. Karypis, Fism: factored item similarity models for top-n recommender systems. In Proceedings of the 19th ACM international conference on Knowledge discovery and data mining, 659-667, 2013.

[4] P. Resnick, N. Iacovou, M. Suchak, P. Bergstrom, and J. Riedl, GroupLens: an open architecture for collaborative filtering of netnews. In Proceedings of the ACM conference on Computer supported cooperative work, 175-186, 1994.

[5] S. Deng, L. Huang, and G. Xu. Social network-based service recommendation with trust enhancement. Expert Systems with Applications, 41, 18 (12/15/), 8075-8084, 2014.

[6] A. Karatzoglou, X. Amatriain, L. Baltrunas, and N. Oliver, Multiverse recommendation: n-dimensional tensor factorization for context-aware collaborative filtering. In 
Proceedings of the fourth ACM conference on

Recommender systems, 79-86, 2010. 